\title{
Spatial heterogeneity of the double-pulse laser plasma of copper alloys
}

\author{
E. Voropay and K. Ermalitskaia ${ }^{a}$ \\ Belarusian State University, Pr. Nezavisimosti, 4, 220030 Minsk, Belarus
}

Received 12 April 2011 / Received in final form 30 June 2011

Published online 11 October 2011 - (c) EDP Sciences, Società Italiana di Fisica, Springer-Verlag 2011

\begin{abstract}
The propagation processes of the plasma excited by double laser pulses in multicomponent copper alloys have been investigated. The methods to study direct and reverse substance deposition from the erosion plume have been developed. The spatial heterogeneity of the brass and bronze ablative plasma composition has been exhibited. With the use of laser beam defocusing relative to the sample surface, the propagation velocities of various elements in the ablative plasma have been estimated.
\end{abstract}

\section{Introduction}

Single- and double-pulse laser-induced breakdown spectroscopy (LIBS) is one of the most promising techniques for qualitative and quantitative atomic emission analysis of different objects [1-4]. Owing to the advantages of laser evaporation and of the ablative plasma excitation, this method has found a wide use for the elemental analysis of natural objects, industrial samples, building materials, objects of art and cultural heritage. One of the most important aspects necessitating further investigation in the process of developing the quantitative analysis methods is the problem of correlation between the ablative plasma composition and that of the starting material.

As demonstrated by the literature data and by the results of our studies, ablation is actually independent of the physical and chemical properties of samples at the flux densities commonly used for analytical purposes $(q=$ $\left.10^{9}-10^{10} \mathrm{~W} / \mathrm{cm}^{2}\right)[3-6]$, although at these parameters the plasma-second pulse interaction may lead to the appearance of spatial and temporal heterogeneities in the propagating ablative plume $[6,7]$. Besides, one should take into account the differences of the local plasma temperature and particle density in various regions of the plume [8]. An analytical signal from the element, the atoms of which are predominantly at the plasma periphery that is relatively colder than the central part, displays conservative values of the concentration. Because of this, spatial heterogeneity of the multicomponent ablative plasma may lead to significant errors in the results of quantitative analysis, especially when using the calibration-free method based on the calculation of Boltzmann expression for the line emissivities [9]. The main objectives of this work are to study the propagation processes of the plasma formed by singleand double-pulse laser ablation of multicomponent copper alloys, to evaluate the radial distribution of elements

\footnotetext{
a e-mail: ermalitskaia@gmail.com
}

in direct- and backward-deposited films, and to estimate the average velocity of different types of atoms in the ablative plume.

\section{Experimental}

The plasma was generated at the fundamental wavelength $(1064 \mathrm{~nm})$ of a nanosecond Q-switched doublepulse Nd:YAG laser (LOTIS, Belarus) with a pulse width of $15 \mathrm{~ns}$ and repetition rate of $10 \mathrm{~Hz}$. Double laser pulses with the interpulse interval $\Delta t=0-100 \mu \mathrm{s}$ (1 $\mu \mathrm{s}$ step) were used to vaporize the sample and to excite the atomic spectra. Investigation of the spatial heterogeneity of the double-pulse laser multicomponent plasma was performed at the interpulse delays and pulse energies most commonly used for analytical purposes. A most significant increase in the spectral line intensities of all the considered elements was observed for $\Delta t=5 \div 15 \mu \mathrm{s}$ and during the experiments the interpulse delay was varied from 1 to $20 \mu \mathrm{s}$. The effect of double laser pulses with zero interpulse interval $(\Delta t=0 \mu \mathrm{s})$ was identical to that of a single pulse with the doubled energy. Laser pulses with the energy ranging from 10 to $70 \mathrm{~mJ}$ were focused by a lens of $100-\mathrm{mm}$ focal length, with the spot diameter on the sample approximating $50-100 \mu \mathrm{m}$. The laser beam was aligned horizontally at right angles to the sample surface and the plasma was generated in the air at atmospheric pressure.

A simulation study of the plasma propagation in the presence of air has been performed by Bogaerts et al. [10] It was shown that at the laser irradiance $10^{9}-10^{10} \mathrm{~W} / \mathrm{cm}^{2}$ the plume pushed background gas away from the target leading to depletion in density near the surface and to the increased gas density in front of the vapor plume. The main difference between ablation in the vacuum and in the presence of background gas is forming of a shock wave moving away from the target.

As suggested by Han et al., the shock wave formation in a buffer gas and the plasma shielding play an important 

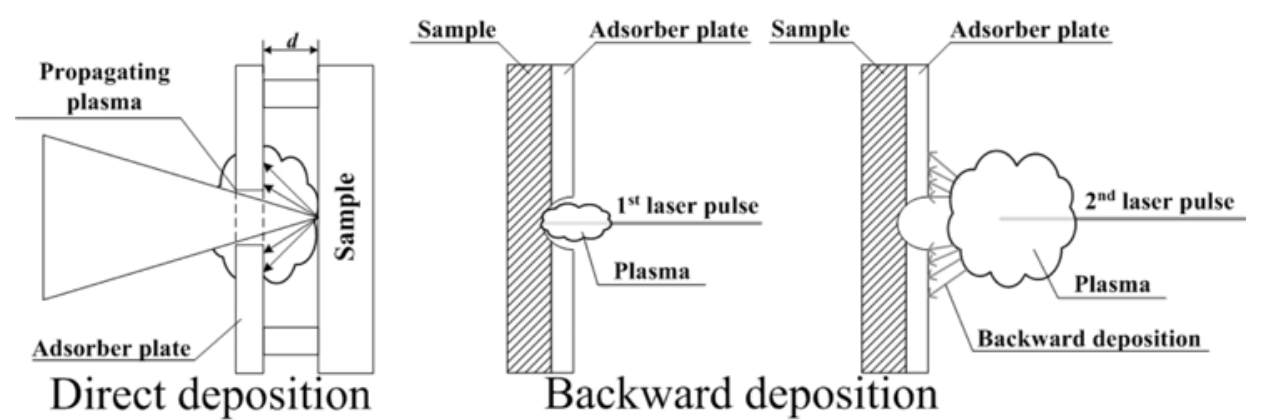

Fig. 1. Schematic of experiments with direct and backward deposition from the ablative plasma excited by double laser pulses.

role in the cluster formation in the plume due to the increasing rate of particle collisions between clusters, vapor atoms, and gas atoms [11]. Brailovsky et al. have shown that the condensation droplets can be formed at laser intensities $10^{8}-10^{10} \mathrm{~W} / \mathrm{cm}^{2}$ and at the pulses of several nanoseconds under slow expansion of the vapor plume into the background gas [12].

The analytical signal was recorded synchronously with the arrival of the second pulse in order to eliminate registration of continuous spectra of the air. The continuum emitted by the leading front of the plasma formed by the second pulse was much weaker because of the reduced particle density of the surrounding atmosphere in the near-surface region. Radiation of the sample plasma was collected by two achromatic objectives into two SDH-1 compact polychromators (SOLAR Laser Systems, Minsk) with the working range $190-340 \mathrm{~nm}$ and $265-800 \mathrm{~nm}$, respectively. In both cases the recorded spectral width $\sim 150 \mathrm{~nm}$; range of the second polychromator was retuned by a microscrew. The SDH-1 polychromator (grating 1200 lines/mm; the average inverse linear dispersion $5.3 \mathrm{~nm} / \mathrm{mm}$; spectral resolution $0.12 \mathrm{~nm}$ ) was a diffraction spectrograph providing a linear spectral scan for the recording system based on a 3648-pixel multi-channel TCD1304AP detector (Toshiba).

Two different copper alloys (brass containing $\mathrm{Cu}-$ $60.92 \% ; \mathrm{Zn}-36.78 \% ; \mathrm{Pb}-1.39 \% ; \mathrm{Sn}-0.15 \% ; \mathrm{Al}-0.39 \%$; $\mathrm{Ni}-0.28 \% ; \mathrm{Fe}-0.09 \%$ and bronze containing $\mathrm{Cu}-86$. $29 \% ; \mathrm{Sn}-11.71 \% ; \mathrm{Zn}-0.75 \% ; \mathrm{Pb}-0.29 \% ; \mathrm{P}-0.96 \%)$ were selected to study the spatial heterogeneity of the multicomponent ablative plasma. The spatial distributions in the ablative plume were examined with the use of the specially developed experimental methods enabling both direct and backward deposition of the material (see Fig. 1).

Aguilera and Aragon have compared the synthesized and experimental intensity distributions of the neutral atom and ion emission lines in the ablative plasma of metals and have found that a maximal intensity was observed at a distance of $1-2 \mathrm{~mm}$ from the target [13]. A maximal intensity corresponds to the highest density of the atoms and ions in the plume. Similar results were presented by Bindhu et al.: 90 ns after the laser pulse the plume length was approximately $5 \mathrm{~mm}$ with the central dense part of $1 \mathrm{~mm}$ [14]. These data were used during the experiments with direct and backward deposition from the ablative plasma. A distance between the target and adsorber plate for backward deposition and a thickness of the adsorber for direct deposition were chosen in order to investigate a deposited film in the plasma region with the highest density.

During the direct deposition experiments an adsorber glass plate was fixed $1 \mathrm{~mm}$ above the surface of a brass or bronze sample. Laser radiation passing through $1 \mathrm{~mm}$ hole in the glass plate vaporized the sample. The central part of the resultant ablative plasma freely propagated through the hole, whereas in the peripheral regions at a distance exceeding $1 \mathrm{~mm}$ from the laser beam axis the layers were deposited on the internal surface of the glass plate. In the backward deposition experiments the glass plate $1 \mathrm{~mm}$ thick was fixed directly on the brass or bronze sample [15]. In this case the deposited film was analyzed on the external surface of the glass plate.

A semi-quantitative analysis of the material deposited on the adsorber plate was performed with the help of a LSS-1 spectrometer. A relative elemental concentration in the deposited films was evaluated as a ratio of the spectral line intensities for different components. When the spectral line intensity of some element was on a background level, it was assumed that atoms of this component were absent in the film or their concentration was under the detection limits of LSS-1 $(C<0.001 \%)$.

\section{Results and discussion}

The performed study of the radial distribution on the adsorber plate after double pulse ablation of brass and bronze has demonstrated that the deposited film is inhomogeneous and the content of the deposited layer is at variance with the concentrations in the original sample. Copper was detected within $5 \mathrm{~mm}$ from the hole, zinc $3 \mathrm{~mm}$ for direct and $4 \mathrm{~mm}$ for backward deposition, lead $-2 \mathrm{~mm}$. The same radial elemental distribution was observed after double pulse ablation of bronze: copper was detected within $5 \mathrm{~mm}$ from the hole; tin - only within a circular region with a radius of $3 \mathrm{~mm}$. The spectral line intensities of the remaining components of copper alloy in the deposited films were on a background level, indicating that their concentrations in the deposited layer were below $0.001 \%$. The obtained results demonstrate that elements with smaller melting enthalpy (tin, lead, aluminum, zinc) of the investigated alloys propagate mostly in a dense central region of the plasma [15]. 

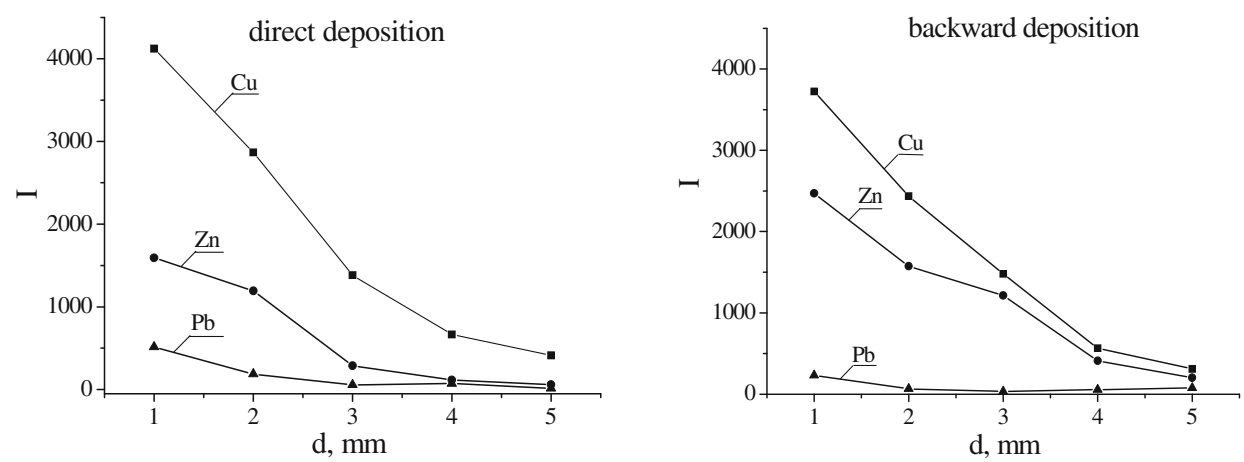

Fig. 2. Spatial distribution of the spectral line intensity of copper $\lambda_{\mathrm{Cu}}=324.754 \mathrm{~nm}$, zinc $\lambda_{\mathrm{Zn}}=334.502 \mathrm{~nm}$, and lead $\lambda_{\mathrm{Pb}}=405.798 \mathrm{~nm}$ in the direct- and backward-deposited film due to double pulse ablation of a brass sample at the pulse energy $100 \mathrm{~mJ}$.

The spectral line intensities of copper $-\lambda_{\mathrm{Cu}}=$ $324.754 \mathrm{~nm}$, zinc $-\lambda_{\mathrm{Zn}}=334.502 \mathrm{~nm}$, and lead $-\lambda_{\mathrm{Pb}}=$ $405.798 \mathrm{~nm}$ are shown in Figure 2 as a function of the distance $d$ from the laser beam axis in direct and backward deposition of the material from the ablative plasma formed due to the effect of double laser pulses with the energy $E_{p}=100 \mathrm{~mJ}$ on brass. As demonstrated by the experiments, an increase in the laser pulse energy leads to growing volume of the deposited material and greater diameter of its spraying on the sample surface. After laser ablation with the pulse energy $50 \mathrm{~mJ}$, zinc is found only within a $3 \mathrm{~mm}$ circular region, whereas growing of the energy to $100 \mathrm{~mJ}$ leads to greater spraying of zinc on the adsorber plate $(4 \mathrm{~mm})$. At the pulse energy $100 \mathrm{~mJ}$ copper is detected within the region of $5 \mathrm{~mm}$, lead $-2 \mathrm{~mm}$; at $30 \mathrm{~mJ}$ the radius of copper deposition is $4 \mathrm{~mm}$, and the lead content on the adsorber plate in this case is under the detection limits being below $0.001 \%$.

The data obtained indicate that the ablative plume is extended along the axis of a laser beam. Elements with smaller melting enthalpy (zinc, lead, tin) expand mainly within a narrow central region of the plasma that passes through the hole in the adsorber plate without deposition. On ablation of brass or bronze, during expansion of the plasma to the diameter that is equal to the hole width in the adsorber plate, the atoms of zinc, lead, and tin have time to leave the region between the plate and the sample surface. This means that the velocity of components with the smaller melting enthalpy along the laser beam is higher than in the perpendicular direction. The situation is somewhat different from copper atoms in the ablative plasma of brass or bronze: velocity along the laser beam is lower than in the perpendicular direction so copper atoms reaching the adsorber plate (1 $\mathrm{mm}$ distance) are located within $4 \mathrm{~mm}$ from the centre of the hole.

The differences between the axial and radial velocities of copper and zinc in the ablative plasma and in the deposited layer on the adsorber (Tab. 1) are supported by the increased ratio of their line intensities compared to the double-pulse ablation of the original brass sample $\left(I_{\mathrm{Cu}} / I_{\mathrm{Zn}} \sim 0.9 \div 1\right)$. This growing of $I_{\mathrm{Cu}} / I_{\mathrm{Zn}}$ indicates that in the direct- and backward-deposited film the concentration of zinc is reduced and the content of copper is increased. The central part of the plasma that propagates
Table 1. Ratios of the spectral line intensities for copper $\lambda_{\mathrm{Cu}}=324.754 \mathrm{~nm}$ and zinc $\lambda_{\mathrm{Zn}}=334.502 \mathrm{~nm}$ in brass deposited on the adsorber on double pulse ablation at different interpulse intervals $\Delta t$.

\begin{tabular}{cccccc}
\hline & \multicolumn{5}{c}{$\Delta t, \mu \mathrm{s}$} \\
\cline { 2 - 6 } & 0 & 1 & 5 & 10 & 15 \\
\cline { 2 - 6 } & \multicolumn{5}{c}{$I_{\mathrm{Cu}} / I_{\mathrm{Zn}}$} \\
\hline 1 & 10 & 7 & 4 & 3 & 3 \\
2 & 17 & 9 & 6 & 5 & 4 \\
3 & - & 13 & 9 & 7 & 6 \\
4 & - & 19 & 13 & 10 & 8 \\
\hline
\end{tabular}

through the hole without deposition reveals the reduced concentration of less volatile copper and increased content of components with smaller melting enthalpy from brass or bronze.

Backward deposition of the particles from the plasma created by single laser pulses $(\Delta t=0 \mu \mathrm{s})$ is governed by the collision frequency, by the probability that an atom receives impetus towards the sample surface, and by the probability that an atom flies through the steam-gas mixture after collision. These factors should be taken into account in physical models for backward deposition. In this way the thickness and composition of deposited films are dependent on the volume of the evaporated material of copper alloys as well as on the temperature, density, and propagation velocity of the plasma. As shown by the experiments, coming back of the particles from the steam-gas mixture to the sample surface is continued for a long time after the laser pulse. The publications devoted to the experimental and theoretical studies of laser ablation point to the fact that almost $20 \%$ of the ablated particles are backward-deposited on the sample $[11,16]$.

The backward particle flux caused by atomic collisions in the ablative plasma is approximately identical for all interpulse intervals $\Delta t$, being determined by the pulse energy only. The number of collisions in the ablative plasma may be evaluated by comparing the thermal velocities $u$ of atoms with the mass $m$ in a saturated steam as (1):

$$
u=\sqrt{\frac{2 k T}{m}}
$$


where $k$ - Boltzmann constant $\left(\mathrm{J} \mathrm{K}^{-1}\right), T$ - plasma temperature $(\mathrm{K})$.

The method used to determine the temperature is based on the assumption that a local thermal equilibrium exists in the laser-induced plasma. Saha and Boltzmann expressions define spectral line intensity as a single-valued function of the plasma temperature (2) [17]:

$$
I=A_{e i} h \nu_{e i} n_{0} \frac{g_{e}}{g_{0}} e^{-\frac{E_{e}}{k T}}
$$

By substitution of the values for the energy, transition probabilities, and degeneracy of the $i$-level for the spectral lines of copper $\lambda_{\mathrm{Cu}}=510.554$ and $515.324 \mathrm{~nm}$ we derive the expression (3) for the plasma temperature:

$$
T=\frac{11945}{1.826+\lg \left(I_{510} / I_{515}\right)},
$$

where $I_{510}$ and $I_{515}$ are intensities of the corresponding spectral lines.

The changes in the plasma temperature $\Delta T$ and thermal velocities $\Delta u$ of the particles were evaluated as (4) using equations (1) and (3)

$$
\begin{gathered}
\Delta T=\frac{T_{\text {double }}-T_{\text {single }}}{T_{\text {single }}} \times 100 \%, \\
\Delta u=\frac{u_{\text {double }}-u_{\text {single }}}{u_{\text {single }}} \times 100 \%,
\end{gathered}
$$

where $T_{\text {single }}$ and $T_{\text {double }}, u_{\text {single }}$ and $u_{\text {double }}$ are the plasma temperature and thermal particle velocities in the ablative plasma formed under the effect of single and double laser pulses.

According to the performed calculations, doubling of the pulse energy (from 50 to $100 \mathrm{~mJ}$ ) leads to a $1-2 \%$ temperature growth. Similar results were described in the book titled "Handbook of Laser-Induced Breakdown Spectroscopy" by Cremers and Radziemski [18]. After the beginning of the first laser pulse, an ultradense cloud of atoms, ions, and electrons occurs in several picoseconds. The leading edge of the forming plasma with a rather high electron density can absorb or reflect light almost completely. As a result, only an insignificant part of the pulse energy can directly interact with the sample. Thus at nanosecond laser pulses, due to the plasma shielding, the pulse energy doubling leads to a very insignificant growth of the signal enhancement. As it was calculated by Bogaerts and Chen and shown by Russo and Mao, the electron density was differently decreased as a function of time $[19,20]$.

The shock wave formed on the leading edge of the plasma ejects the surrounding gas away from the sample surface. According to Scaffidi, the main reason for the signal enhancement in a double pulse configuration is the substance evaporation by the second pulse to the nearsurface region at high temperatures and low gas densities [21]. Because of this, shielding of the laser energy by the leading edge of the plasma created by the second pulse is reduced. A greater part of the energy is spent to heating of the sample surface and substance evaporated by the first pulses and remaining in the near-surface region.

Thus, the energy doubling due to shielding by the leading edge of the plasma leads to a very insignificant temperature growth. The use of sequential delayed pulses causes notable heating due to changed conditions in the nearsurface region when the second pulse arrives.

On going from single to double laser pulses at the fixed total energy and power the plasma temperature change is increased by $10-15 \%$, whereas the thermal velocity in this case is growing by $6-7 \%$. An increase of the interpulse interval $\Delta t$ for more than $1 \mu$ s results in a gradual reduction of the temperature and thermal velocity differences between single- and double-pulse laser ablation. In this way an increase of the plasma temperature in the doublepulse configuration leads to greater thermal velocities of the particles and hence to more collisions in the plasma.

The cause for the increased backward flux from the plasma to the sample surface due to radiation of the second pulse was investigated by a quantitative analysis of the multicomponent film deposited at different distances from the laser beam axis. It was found that a change in the interpulse interval $\Delta t$ leads to changes in the plasma-laser pulse interaction at different stages of the plasma propagation, and also to changes in the quantity and composition of the material deposited on the adsorber plate. Owing to the performed experiments, it was demonstrated that on going from single- to double-pulse ablation a multiple growth of the backward flux of brass ( 5 times for copper and 3.5 times for zinc) and bronze (4.5 times for copper and 2.5 times for tin) is observed. Both with single- and double-pulse ablation, the multicomponent plasma created as a result of single- or double-pulse ablation features spatial heterogeneity: elements with smaller melting enthalpy (zinc, tin, lead) spread at higher velocities in the central "hot" region of the plume, whereas the elements with higher melting enthalpy are mostly localized at the plasma periphery.

The spatial heterogeneity of the ablative plume formed during double-pulse laser ablation of copper alloys is also confirmed by calculations of the plasma temperature by relative intensities of spectral lines using Boltzmann plot. The line integral intensity $I_{i j}$, corresponding to the transition between two levels $i$ and $j$ with the energies $E_{\mathrm{i}}$ and $E_{j}$, is determined by the relation (5) [17]:

$$
I_{i j}=F C A_{i j} \frac{g_{i} e^{-\frac{E_{i}}{k T}}}{U(T)},
$$

where $C$ is the concentration of the emitting atomic species, $A_{i j}$ is the transition probability, $g_{i}$ degeneracy of the $i$-level; $k$ Boltzmann constant; $T$ is plasma temperature, $F$ experimental parameter taking into account the optical efficiency of the system used and some plasma parameters. The distribution function $U(T)$ of the $i$-th atom is given by (6)

$$
U(T)=\sum_{i} g_{i} \exp \left(-E_{i} / k T\right) .
$$



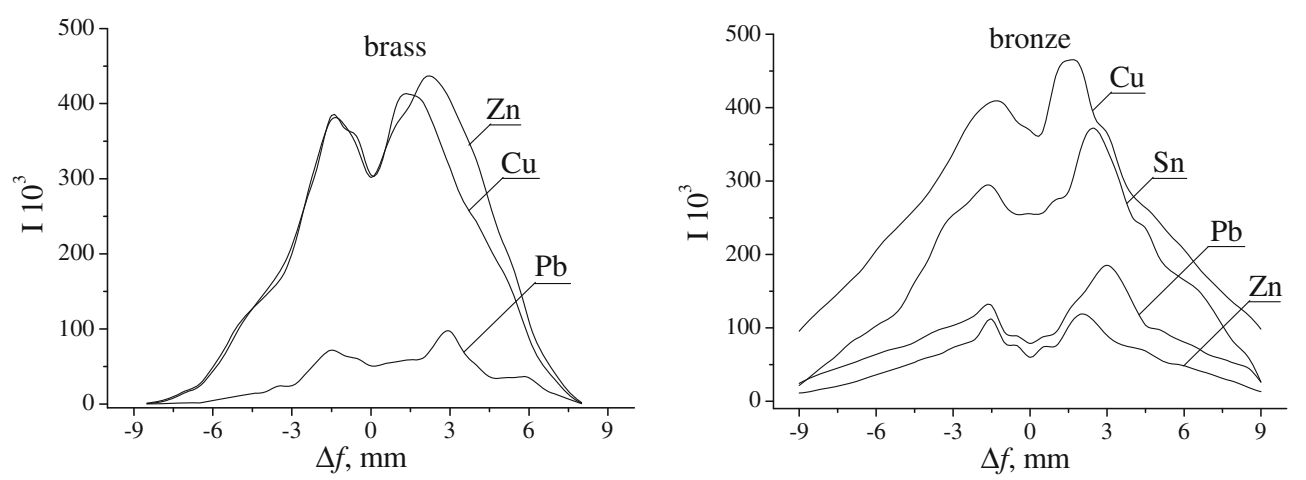

Fig. 3. Spectral line intensities of copper $\lambda_{\mathrm{Cu}}=324.754 \mathrm{~nm}$, tin $\lambda_{\mathrm{Sn}}=326.233 \mathrm{~nm}$, zinc $\lambda_{\mathrm{Zn}}=334.502 \mathrm{~nm}$, and lead $\lambda_{\mathrm{Pb}}=405.782 \mathrm{~nm}$ as a function of the defocusing parameter $\Delta f$.

Taking the logarithm of expression (6) and using the definitions (7)

$$
y=\ln \frac{I_{i j}}{g_{i} A_{i j}}, \quad m=-\frac{1}{k T}, \quad x=E_{i}, \quad p=\ln \frac{C F}{U(T)},
$$

we derive a linear relationship (8) between $x$ and $y$

$$
y=m x+p
$$

According to (8), the values of $y$ corresponding to the intensity of different lines from the same emitting species will be distributed linearly with respect to the energy of the upper transition level; the slope of the distribution curve being related to the plasma temperature. On the assumption that the ablative plasma remains at the local thermodynamic equilibrium (LTE), after determining the plasma temperature, the species concentrations can be obtained by the normalization relation (9) since a sum of the relative concentrations of all the elements must be unity [22]

$$
\sum_{k} C_{k}=1
$$

When the plasma temperature is known, it is possible to determine the concentration of the element of interest by measuring just one experimental emission line with the known parameters $E_{i}, A_{i j}, g_{i}$. Applying this method to determine a temperature of the double pulse laser-induced plasma, we have obtained distinct results for the calculations performed using the spectral line intensities of different components: copper $-9400 \mathrm{~K}$; tin $-7500 \mathrm{~K}$; lead - $6700 \mathrm{~K}$, and zinc $-8700 \mathrm{~K}$. The obtained data indicate that atoms of different species are predominantly localized in different regions of the propagating plasma, i.e. the components of copper alloys in the ablative plume are differing in their recession velocities.

Recession velocities of brass and bronze components in the plasma were evaluated in the experiments with positive and negative defocusing of the laser beam - focusing at some distance above and below the surface of the ablated sample [23]. An increase in the spectral line intensity of all the components of brass and bronze was observed in both cases (Fig. 3).
A maximal intensity of the spectral lines for all components of copper alloys after single pulse ablation was observed at $\Delta f= \pm 0.5 \mathrm{~mm}$. Aguilera and Aragon showed similar radial distribution of the plasma temperature, and also analogous electron and ion densities as a function of the distance to the laser beam axis [24]. The observed dip in the spectral line intensities is caused by self-absorption and shielding of laser radiation by the dense plasma. Reduction of the flux density $q$ after defocusing leads to a decrease in the amount of evaporated material and in the density of the propagating steam-gas mixture. At small distances $\Delta f$ almost all radiation of the second laser pulse is transmitted without absorption to the surface of the sample and almost all the energy is spent on evaporation. Further defocusing leads to reduction of the flux density with simultaneous growing of the portion of laser energy spent on heating and melting of the sample rather than ablation. The same dependence was observed for the defocused double pulse ablation of bronze [23].

At small distances $\Delta f$ an increase of the spectral line intensities for all the elements studied was higher in the case of positive defocusing, indicating that the observed growth of the analytical signal at $\Delta f>0 \mathrm{~mm}$ is caused not only by the reduced shielding of laser radiation but also by the interaction between the second pulse and the plasma formed by the first pulse. And the value of $\Delta f_{\max }$ corresponding to the maximal spectral line intensities is differing for different elements: $\mathrm{Cu}-1.5 \mathrm{~mm}$; $\mathrm{Zn}-2 \mathrm{~mm}$; $\mathrm{Sn}-2.5 \mathrm{~mm} ; \mathrm{Pb}-3 \mathrm{~mm}$. As demonstrated by the data obtained with the interpulse interval $\Delta t=10 \mu \mathrm{s}$, a maximal concentration of the atoms for different elements is observed at different distances from the sample surface. When this distance of the maximal concentration $\Delta f_{\max }$ for one of the components is coincident with the defocusing length $\Delta f$, these atoms acquire an additional excitation due to the second-pulse emission. The schematic of the interaction between the second defocused laser pulse and copper or zinc atoms evaporated by the first laser pulse are shown in Figure 4.

The average expansion velocity $v$ of the elements can be evaluated from the known values of the distance $\Delta f_{\max }$ at the interpulse interval $\Delta t=10 \mu$ s as (10):

$$
\Delta f_{\max }=v \Delta t
$$




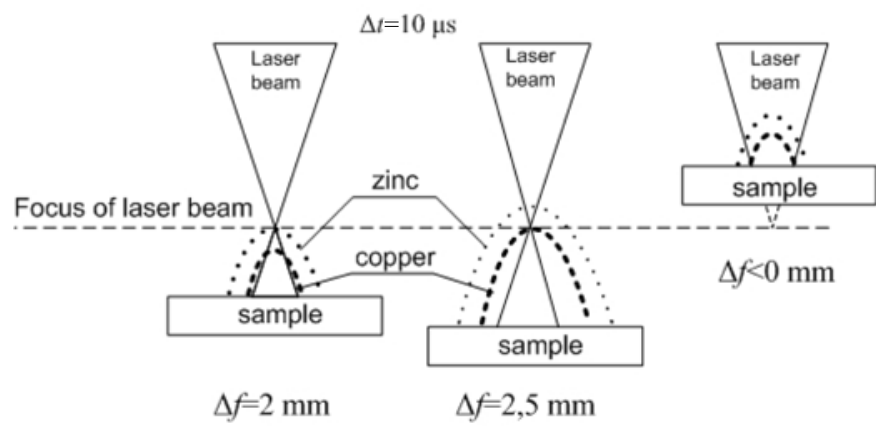

Fig. 4. Schematic of the interaction between the second defocused laser pulse and copper or zinc atoms evaporated by the first laser pulse.

According to equation (10), at the pulse energy $100 \mathrm{~mJ}$ and interpulse interval $\Delta t=10 \mu \mathrm{s}$ the average expansion velocities in the propagating plasma of bronze are: copper - $150 \mathrm{mps}$, zinc - $200 \mathrm{mps}$, tin - $250 \mathrm{mps}$, lead - $300 \mathrm{mps}$. The calculated average velocity of the atoms evaporated by double laser pulses is less by an order of magnitude than that of the ablative plasma. A decrease of the laser pulse energy leads to reduction of the flux density $q$, self-absorption in the plasma, and average velocity of the elements. The performed experiments show that $v$ values depend on the melting enthalpy of the evaporated material, being independent of the atomic mass. A mass of a copper atom (63.5 a.m.u.) is almost equal to the mass of zinc atom (65.4 a.m.u.), but the melting enthalpy of copper is two times greater than that of zinc: 13.0 and $7.2 \mathrm{~kJ} /$ mole, respectively. If the average velocity of a component in the plasma is depending on the atomic mass only, then the particles of copper and zinc propagate at the same velocity.

The observed species segregation is stipulated by the particle formation in the expanding plasma due to the laser-solid interaction [25]. Elemental fractionation in the plume is confirmed by numerous investigations in the field of laser ablation - inductively coupled plasma - mass spectrometry [26]. Spatial heterogeneity of the plasma is caused mainly by preferential volatilization of lighter elements coupled with preferential condensation in the expanding plume [27]. The majority of models describe the particle formation and growth in the plasma based on effusion, condensation, and nucleation theories. Fantoni et al. have demonstrated that partial vapor pressures of the elements in the plume formed after laser ablation of the multicomponent bronzes change differently with time [28]. The pressure of copper species drops down by 4 orders of magnitude in 50 ns after the laser pulse, whereas the pressure of zinc decreases by an order only.

\section{Conclusions}

The performed experiments with a direct and backward deposition from the ablation plasma of brass and bronze and also investigation of double-pulse ablation of copper alloys by the defocused laser beam have demonstrated spatial heterogeneity of the multicomponent ablative plasma.
The atoms of components with smaller melting enthalpy propagate with higher velocities in the central hot part of the plume, whereas the atoms of relatively less volatile copper are localized mostly at the plasma periphery. The differences in the expansion velocities, plasma temperatures in different regions of the plume, and also spatial heterogeneity of the plasma may result in a significant increase of errors in the results of quantitative analysis of copper alloys, especially when using the calibration-free method.

\section{References}

1. Laser-Induced Breakdown Spectroscopy (LIBS): Fundamentals and Applications, edited by A.W. Miziolek, V. Palleschi, I. Schechter (Cambridge University Press, 2006)

2. C. Gautier et al., Spectrochim. Acta, Part B: At. Spectrosc. 61, 210 (2006)

3. G. Cristoforetti et al., Spectrochim. Acta, Part B: At. Spectrosc. 63, 312 (2008)

4. M. Corsi et al., Appl. Geochem. 21, 748 (2006)

5. F. Colao et al., Spectrochim. Acta, Part B: At. Spectrosc. 57, 1167 (2002)

6. K. Novotny et al., Chem. Listy 102, s1309 (2008)

7. S. Laville et al., Spectrochim. Acta, Part B: At. Spectrosc. 64, 1167 (2009)

8. V.S. Piscitelli et al., Spectrochim. Acta, Part B: At. Spectrosc. 64, 147 (2009)

9. E. Tognoni et al., Spectrochim. Acta, Part B: At. Spectrosc. 65, 1 (2010)

10. A. Bogaerts et al., Spectrochim. Acta, Part B: At. Spectrosc. 60, 1280 (2005)

11. M. Han et al., Phys. Lett. A 302, 182 (2002)

12. A.B. Brailovsky, S.V. Gaponov, V.I. Luchin, Appl. Phys. A 76, 339 (1995)

13. J.A. Aguilera, C. Aragon, J. Phys. 59, 210 (2007)

14. C.V. Bindhu et al., Appl. Spectrosc. 58, 719 (2004)

15. K.F. Ermalitskaia, Uzhhorod Univ. Sci. Herald. Series Phys. 24, 51 (2009)

16. C. Aragón, J.A. Aguilera, Spectrochim. Acta, Part B: At. Spectrosc. 63, 893 (2008)

17. T.E. Itina et al., J. Appl. Phys. 83, 6050 (1998)

18. D.A. Cremers, L.J. Radziemski, Handbook of LaserInduced Breakdown Spectroscopy (John Wiley \& Sons Ltd, West Sunssex, 2006)

19. A. Bogaerts, Z. Chen, J. Anal. At. Spectrom. 19, 1169 (2004)

20. X. Mao, R.E. Russo, Appl. Phys. A 64, 1 (1997)

21. J. Scaffidi, S.M. Angel, D.A. Cremers, Anal. Chem. 78, 24 (2006)

22. A. Ciucci et al., Appl. Spectrosc. 53, 960 (1999)

23. K.F. Ermalitskaia et al., J. Appl. Spectrosc. 77, 153 (2010)

24. J.A. Aguilera, C. Aragon, Spectrochim. Acta, Part B: At. Spectrosc. 62, 378 (2007)

25. A. Bogaerts et al., Spectrochim. Acta, Part B: At. Spectrosc. 58, 1867 (2003)

26. S.E. Jackson, D. Gunther, J. Anal. At. Spectrom. 18, 205 (2003)

27. I. Rodushkin et al., J. Anal. At. Spectrom. 17, 831 (2002)

28. R. Fantoni et al., Spectrochim. Acta, Part B: At. Spectrosc. 60, 1186 (2005) 